\title{
Comparación de tecnologías de oxidación (TAO’s) para la degradación del pesticida Mertect
}

\author{
Joan Amir Arroyave Rojas', Luis Fernando Garcés Giraldo², \\ Andrés Felipe Cruz Castellanos ${ }^{3}$
}

1. Docente e Investigador del grupo de investigación Ambiente y Hábitat, Facultad de Arquitectura e Ingeniería, Institución Universitaria Colegio Mayor de Antioquia.

2. Director de Investigaciones, Decano Facultad de Ingenierías, Corporación Universitaria Lasallista, Grupo de Investigación GAMA.

3. Ingeniero Ambiental, Corporación Universitaria Lasallista, Grupo de Investigación GAMA.

Correspondencia: joan.arroyave@colmayor.edu.co

Recibido: 15/04/2011 - Aceptado: 17/08/2011

\begin{abstract}
Resumen
El presente trabajo tuvo como objetivo realizar la comparación de tecnologías avanzadas de oxidación (TAO's) para la degradación del pesticida Mertect. Para el diseño experimental del trabajo se empleó un fotoreactor. La degradación del pesticida se determinó mediante espectrofotometría ultravioleta/visible, método que permitió determinar que los procesos fotoquímicos basados en la fotocatálisis heterogénea con dióxido de titanio - $\mathrm{TiO}_{2}$ y los procesos de fotocatálisis homogénea con hierro (III) permiten obtener porcentajes de remoción significativos del pesticida superiores al $99.0 \%$ y al $95.0 \%$ respectivamente, lo que permite concluir que los procesos de oxidación avanzados son adecuados para la remoción y degradación del pesticida Mertect.
\end{abstract}

Palabras clave: fotodegradación, fotocatálisis homogénea, fotocatálisis heterogénea, aguas residuales, pesticida, tiabendazol, Mertect, industria bananera.

\section{Abstract \\ Comparison of oxidation technologies (TAO's) for pesticide degradation Mertect}

This study aimed to make the comparison of advanced technologies to oxidation (TAO's) to Mertect pesticide degradation. For the experimental design of a photoreactor employment work. Pesticide degradation was determined by spectrophotometry UV / visible, the method revealed that the photochemical processes based on heterogeneous photocatalysis with titanium dioxide - $\mathrm{TiO} 2$ photocatalysis and homogeneous processes with iron (III) allow significant removal rates of the pesticide greater than $99.0 \%$ and $95.0 \%$ respectively, leading to the conclusion that advanced oxidation processes are suitable for the removal and degradation of the pesticide Mertect.

Key words: photodegradation, photocatalysis, wastewater, pesticide, Mertect, thiabendazole, banana industries. 


\section{Introducción}

En la actualidad, se encuentra en desarrollo la aplicación de los procesos avanzados de oxidación (PAOs) que se basan en tecnologías de destrucción de los contaminantes por medio de sustancias químicas conocidas como radicales libres hidroxilos, las cuales tienen la propiedad de ser altamente oxidantes. Dichos radicales reaccionan con el contaminante y lo transforman en compuestos inocuos al ambiente. Para el caso especifico del pesticida Mertect se han estudiado varios procesos avanzados de oxidación entre los cuales se encuentra la oxidación química, fotolisis, fotocatálisis heterogénea y homogénea, buscando degradar dicho contaminante y de esta forma disminuir los impactos negativos que este puede generar en el medio ambiente $(1,2)$.

En la práctica, la aplicación de los métodos de tratamiento debe tener en cuenta fundamentalmente la naturaleza y las propiedades fisicoquímicas de las aguas o efluentes a tratar (3). Las aguas contaminadas por la actividad humana pueden, en general, ser procesadas eficientemente por plantas de tratamiento biológico, por adsorción con carbón activado u otros adsorbentes o por tratamientos químicos convencionales (oxidación térmica, cloración, ozonización, permanganato de potasio, etc.). Sin embargo, en algunos casos estos procedimientos resultan inadecuados para alcanzar el grado de pureza requerido por la legislación o por el uso ulterior del efluente tratado. En estos casos y cada vez más frecuentemente se está expandiendo el uso de las llamadas Tecnologías o Procesos Avanzados de Oxidación (TAO's, PAO's) (1,3). La mayoría de las TAO's puede aplicarse a la remediación y destoxificación de aguas especiales (3-6) generalmente en pequeña o mediana escala. Los métodos pueden usarse solos o combinados entre ellos o con métodos convencionales pudiendo ser aplicados también a contaminantes de aire y suelos permitiendo incluso la desinfección por inactivación de bacterias y virus.

Las TAO's se basan en procesos fisicoquímicos capaces de producir cambios profundos en la estructura química de los contaminantes que involucran la generación y uso de especies transitorias poderosas principalmente el radical hidroxilo
$(\mathrm{HO} \bullet)$. Este radical puede ser generado por medios fotoquímicos (incluida la luz solar) o por otras formas de energía con alta efectividad para la oxidación de materia orgánica. Algunas TAO's, como la fotocatálisis heterogénea, la radiólisis y otras técnicas avanzadas, recurren además a reductores químicos que permiten realizar transformaciones en contaminantes tóxicos poco susceptibles a la oxidación, como iones metálicos o compuestos halogenados (1-3, 5, 7-21).

El proceso de destoxificación mediante fotocatálisis consiste en la utilización de la radiación ultravioleta la cual es muy energética, dicha radiación activa un semiconductor como el dióxido de titanio $\left(\mathrm{TiO}_{2}\right)_{\mathrm{o}}$ el hierro (III) (3, 4, 7-21) para provocar una serie de reacciones de reducción y oxidación, en las que se forma el radical libre hidroxilo que es la especie oxidante primaria formada por la descomposición del peróxido de hidrógeno catalizada por la activación del $\mathrm{TiO}_{2}$ ó el $\mathrm{Fe}^{3+}$. El radical libre hidroxilo es el segundo agente oxidante después del flúor $(\mathrm{HO} \bullet, \mathrm{Eo}=-2,8 \mathrm{~V}$ vs. flúor, $\mathrm{Eo}=-3,0 \mathrm{~V})(3-21)$ y es capaz de realizar oxidaciones no específicas de algunos compuestos orgánicos. Cuando se genera una concentración suficiente de radical libre hidroxilo y otros radicales las reacciones de oxidación de los compuestos orgánicos pueden llegar hasta una total mineralización [9].

Para el caso del presente estudio se evaluaron diferentes tecnologías avanzadas de oxidación buscando establecer las mejores combinaciones de los factores experimentales para la degradación del tiabendazol (Mertect 20 SL) (7,8,15-20), un fungicida empleado ampliamente en la agroindustria bananera para el control del complejo de hongos que genera la pudrición de la corona, del cuello y de los dedos del banano y plátano $(22-28)$.

\section{Materiales y métodos}

Para el desarrollo experimental se emplearon dos de las tecnologías de oxidación avanzada (fotocatálisis heterogénea $\left(\mathrm{TiO}_{2}\right)$ y homogénea $\left(\mathrm{Fe}^{3+}\right)$ basada en la utilización de una lámpara de luz ultravioleta que contiene una cámara donde se hace pasar el afluente del agua residual, allí se realiza la irradiación del agua contaminada con los rayos de luz ultravioleta, se contó 
con una cuba de vidrio que servía de reservorio para el almacenamiento de la muestra de agua. Además, de una bomba sumergible para realizar la recirculación del agua residual por la lámpara UV. El sistema de bombeo se trabajo a un caudal de $0.05 \mathrm{~L} / \mathrm{s}$, para la fotocatálisis homogénea y heterogénea se trabajó con un pH de la solución de 3,0 y 5,0, respectivamente. Se utilizó una concentración inicial de $250 \mathrm{mg} / \mathrm{L}$ del pesticida Mertect concentración típica de las descargas de los desechos líquidos de la industria bananera. La degradación de éste se determinó mediante el empleo de la espectrofotometría UV/Visible.

El diseño experimental utilizado en la investigación fue un diseño factorial de tres factores (tipo de catalizador, concentración de catalizadores y agente oxidante - peróxido de hidrogeno), completamente aleatorizado. Los niveles de cada uno de los factores se encuentran consignados en la Tabla 1 . Adicionalmente, se realizaron tres replicas por corrida experimental, al final de los ensayos se determino la remoción del pesticida mediante el empleo de la espectrofotometría UV/Visible a una longitud de onda de $425 \mathrm{~nm}$.

El modelo estadístico utilizado para el análisis de la información fue:

$$
\begin{aligned}
& Y_{\mathrm{ijkl}}=\mu+\alpha_{\mathrm{i}}+\beta_{\mathrm{j}}+\chi_{\mathrm{k}}+(\alpha \beta)_{\mathrm{ij}}+(\alpha \chi)_{\mathrm{ik}}+(\beta \chi)_{\mathrm{jk}}+ \\
& (\alpha \beta \chi)_{\mathrm{ijk}}+\varepsilon_{\mathrm{ijkl}}
\end{aligned}
$$

En donde,

$\mu=$ Es el porcentaje medio de remoción o degradación del pesticida mertec en el modelo.

$\alpha_{i}=$ Es el efecto del i-ésimo nivel del tipo de catalizador (fotolisis, dióxido de titanio, hierro).

$\beta_{\mathrm{j}}=$ Es el efecto del $\mathrm{j}$-ésimo nivel del factor concentración de catalizador (dióxido de titanio; $\mathrm{i}=0,50$ y $100 \mathrm{mg} / \mathrm{L}-$ hierro (III); $\mathrm{i}=0,25$ y $50 \mathrm{mg} / \mathrm{L})$.

$\chi_{\mathrm{k}}=$ Es el efecto del k-ésimo nivel del factor concentración de agente oxidante - peróxido de hidrogeno; $j$ = 0, 1 y $2 \% \mathrm{v} / \mathrm{v}$.

$(\alpha \beta)_{\mathrm{ij}}=$ Es el efecto de la interacción entre los niveles i y j de los factores tipo de catalizador y la concentración de catalizador.

$(\alpha \chi)_{\text {ik }}=$ Es el efecto de la interacción entre los niveles i y $\mathrm{k}$ de los factores tipo de catalizador y la concentración de agente oxidante - peróxido de hidrogeno. $(\beta \chi)_{\mathrm{jk}}=$ Es el efecto de la interacción entre los niveles j y k de los factores concentración de catalizador y la concentración de agente oxidante - peróxido de hidrogeno.

$(\alpha \beta \chi)_{\mathrm{ijk}}=$ Es el efecto de la interacción triple entre los niveles i, j y k de los factores tipo de catalizador, concentración de catalizador y la concentración de agente oxidante - peróxido de hidrogeno.

$\varepsilon_{\mathrm{ijkl}}=$ Es el l-ésimo termino de error aleatorio.

\section{Resultados}

En la Tabla 1 y la Figura 1 se muestran de forma comparativa los porcentajes de degradación del Mertect de acuerdo con las combinaciones planteadas en el diseńo experimental donde se variaron el tipo de catalizador, las concentraciones catalizador (dióxido de titanio y del hierro (III)) como del agente oxidante (peróxido de hidrógeno). De esta forma se pudo identificar que el pesticida Mertect es un contaminante medianamente fotoactivo. En esta investigación se alcanzaron remociones del 49,3\% del pesticida.

En la Figura 1 se observa que los procesos de degradación fotoquímicos, donde se combinaron diferentes concentraciones de catalizadores y agente oxidante se obtuvieron altos porcentajes de remoción del pesticida Mertect lo que permite concluir que los procesos fotoquímicos son adecuados para su remoción.

El análisis estadístico de los datos experimentales, empleando el ANOVA, determino que para los factores tipo de agente catalizador, el agente oxidante (peróxido de hidrógeno) las interacciones dobles que existen entre los tres factores y la interacción triple entre los factores experimentales poseen una variación significativa en la variable respuesta, es decir, en el

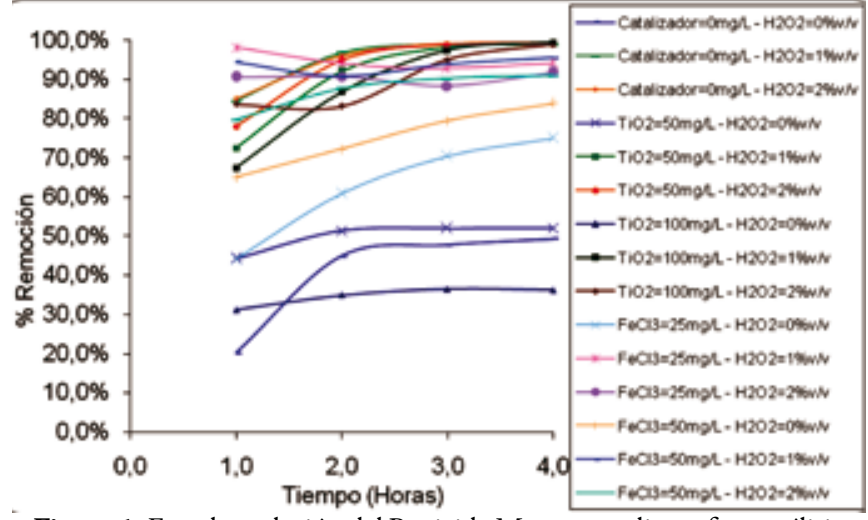

Figura 1. Fotodegradación del Pesticida Mertect mediante fotocatálisis. 
Tabla1. Combinaciones experimentales de factores y porcentajes de remoción $(6,7)$.

\begin{tabular}{|c|c|c|c|}
\hline TAO's & $\begin{array}{c}\text { Concentración Catalizador } \\
(\mathbf{m g} / \mathrm{L})\end{array}$ & $\mathrm{H}_{2} \mathrm{O}_{2}(\% \mathrm{v} / \mathrm{v})$ & \% Remoción \\
\hline Fotolisis & 0,0 & 0,0 & $49,30 \%$ \\
\hline \multirow[b]{2}{*}{ Oxidación Química } & 0,0 & 1,0 & $98,80 \%$ \\
\hline & 0,0 & 2,0 & $98,90 \%$ \\
\hline \multirow{6}{*}{$\begin{array}{l}\text { Fotocatálisis Heterogénea } \\
\qquad\left(\mathrm{TiO}_{2}\right)\end{array}$} & 50,0 & 0,0 & $52,10 \%$ \\
\hline & 50,0 & 1,0 & $99,50 \%$ \\
\hline & 50,0 & 2,0 & $99,40 \%$ \\
\hline & 100,0 & 0,0 & $36,20 \%$ \\
\hline & 100,0 & 1,0 & $99,50 \%$ \\
\hline & 100,0 & 2,0 & $99,00 \%$ \\
\hline \multirow{6}{*}{$\begin{array}{l}\text { Fotocatálisis Homogénea } \\
\text { (Hierro (III)) }\end{array}$} & 25,0 & 0,0 & $75,00 \%$ \\
\hline & 25,0 & 1,0 & $94,20 \%$ \\
\hline & 25,0 & 2,0 & $93,10 \%$ \\
\hline & 50,0 & 0,0 & $83,90 \%$ \\
\hline & 50,0 & 1,0 & $95,60 \%$ \\
\hline & 50,0 & 2,0 & $91,00 \%$ \\
\hline
\end{tabular}

Tabla 2. Análisis estadístico ANOVA de los datos experimentales.

\begin{tabular}{|c|c|c|c|c|c|}
\hline Fuente & $\begin{array}{c}\text { Grados de } \\
\text { Libertad }\end{array}$ & $\begin{array}{c}\text { Suma de } \\
\text { Cuadrados }\end{array}$ & $\begin{array}{c}\text { Promedio al } \\
\text { Cuadrado }\end{array}$ & Coeficiente F & Valor de p \\
\hline A: Tipo de Catalizador & 1 & 260,48 & 260,48 & 11,93 & 0,0014 \\
\hline $\begin{array}{l}\text { B: Concentración de } \\
\text { Catalizador }\end{array}$ & 2 & 48,06 & 24,03 & 1,10 & 0,3435 \\
\hline C: Agente Oxidante & 2 & 18280,70 & 9140,33 & 418,80 & 0,0000 \\
\hline \multicolumn{6}{|l|}{ Interacciones } \\
\hline $\mathrm{AB}$ & 2 & 392,70 & 196,35 & 9,00 & 0,0007 \\
\hline $\mathrm{AC}$ & 2 & 2470,89 & 1235,45 & 56,61 & 0,0000 \\
\hline BC & 4 & 759,43 & 189,86 & 8,70 & 0,0001 \\
\hline $\mathrm{ABC}$ & 4 & 1463,35 & 365,84 & 16,76 & 0,0000 \\
\hline Residuos & 36 & 785,70 & 21,83 & & \\
\hline Total (Corregido) & 53 & 24461,30 & & & \\
\hline
\end{tabular}

porcentaje de remoción del pesticida Mertect, el modelo es significativo estadísticamente debido a que el error máximo permisible o aceptado por el ANOVA para el modelo estadístico es del 5\%. Los resultados obtenidos del modelo empleado, sirve para explicar la degradación del pesticida Mertect de acuerdo a los factores definidos, debido a que el Valor de p es inferior a 0.05 , Tabla 2 .

La Figura 2 presenta el método utilizado para identificar las diferencias significativas de Fisher (LSD), en las que se observa la variación del tipo de catalizador empleado en la fotodegradación del pesticida Mertect entre el (1) dióxido de titanio y (2) el hierro con una diferencia estadísticamente significativa a un nivel de confianza 95,0\%.

De esta misma forma en la Figura 3, se observa que para los intervalos de los niveles del agente oxidante - peróxido de hidrógeno con un nivel de confianza del 95\%, las variaciones de las medias entre $0-1 \%$ v/v y $0-2 \%$ v/v de peróxido de hidrógeno se observan diferencias significativas entre los niveles experimentales, mientras que para la variación entre $1-2 \%$ v/v de peróxido no se observa una variación significativa entre estos dos niveles.

Se identificó la mediana fotoactividad del pesticida y se evidencio que el empleo de peróxido de hidrógeno y agentes catalizadores como el dióxido de titanio y el hierro (III) favorecen las reacciones de degradación del mismo. Esto último se comprueba de acuerdo al análisis estadístico del ANOVA en donde se encuentran diferencias significativas entre los diferentes niveles de los factores y entre ellos mismos. 


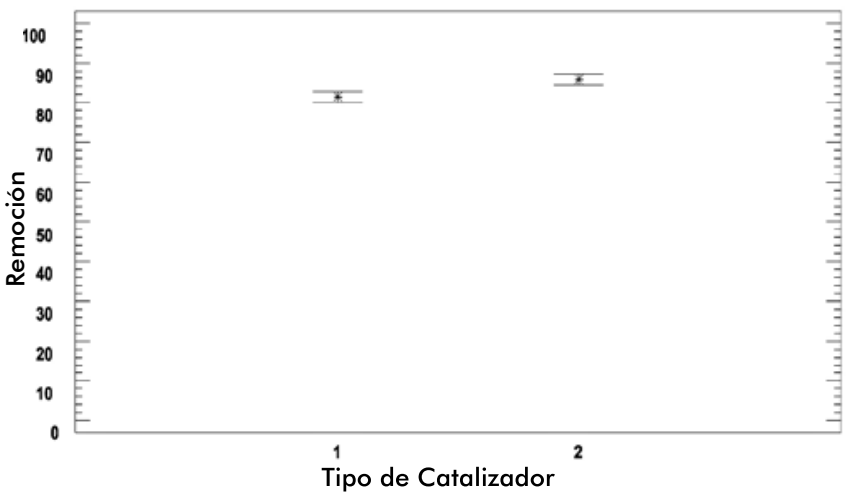

Figura 2. Análisis de medias para intervalos de confianza 95\% LSD para el tipo de catalizador: (1) dióxido de titanio, (2) hierro (III).

\section{Discusión}

De acuerdo a los resultados obtenidos durante este trabajo se encontró que el pesticida Mertect se puede eliminar eficientemente por oxidación química, mediante el empleo de peróxido de hidrógeno, alcanzando porcentajes de remoción superiores al 98\% del pesticida.

Por otra parte, se determinaron altos porcentajes de remoción para el pesticida mediante combinaciones experimentales que representan oxidaciones químicas, utilizando agentes oxidantes, como es el caso de peróxido de hidrógeno en combinaciones de $0 \mathrm{mg} / \mathrm{L}$ de catalizador y para las concentraciones de $1 \% \mathrm{v} / \mathrm{v}$ y $2 \% \mathrm{v} / \mathrm{v}$ de peróxido de hidrógeno con remociones del 98,80 y $98,90 \%$ respectivamente.

El mayor porcentaje para el proceso de fotocatálisis homogénea se presentó para la combinación de $50 \mathrm{mg} / \mathrm{L}$ de hierro (III) y una concentración de $1 \% \mathrm{v} / \mathrm{v}$ de peróxido de hidrógeno con una remoción del $95,6 \%$, mientras que para el proceso de la fotocatálisis heterogénea se presentó en las combinaciones experimentales de $50 \mathrm{mg} / \mathrm{L}$ de dióxido de titanio y una concentración de $1 \% \mathrm{v} / \mathrm{v}$ de peróxido de hidrógeno y $100 \mathrm{mg} / \mathrm{L}$ de dióxido de titanio y una concentración de $1 \% \mathrm{v} / \mathrm{v}$ de peróxido de hidrógeno con una remoción del 99,5\%.

De esta forma la fotocatálisis heterogénea alcanzó porcentajes de remoción del pesticida Mertect más elevados que la fotocatálisis homogénea. Sin embargo, a lo largo del desarrollo experimental se obtuvieron mejores porcentajes de remoción para las primeras horas de experimentación para el segundo proceso, lo cual podría indicar que se podría plantear

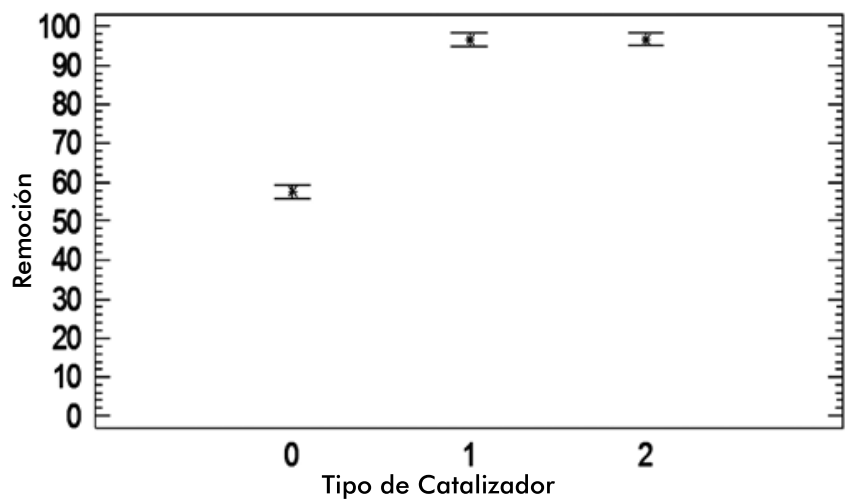

Figura 3. Análisis de medias para intervalos de confianza 95\% LSD para el agente oxidante (peróxido de hidrógeno).

la realización de una combinación entre estos dos procesos de oxidación avanzada en la cual se comience con un procesos homogéneo como lo es la fotofenton y se culmine con un proceso heterogéneo con dióxido de titanio.

Se observó que la fotocatálisis heterogénea y homogénea empleando catalizadores ambientalmente inocuos, las cuales hacen parte de las tecnologías avanzados de oxidación (TAO's), son procesos eficientes para la remoción y degradación de pesticida Mertect. Estas tecnologías podrían contribuir al mejoramiento de los desequilibrios ambientales que generan este tipo de pesticidas en el ambiente.

Finalmente, se observó como los procesos avanzados de oxidación, en este caso, la reacción de fotofenton y la fotocatálisis heterogénea con dióxido de titanio son tecnologías apropiadas para lograr la degradación de compuestos recalcitrantes y persistentes como es el caso del pesticida Mertect contribuyendo a la disminución de los impactos negativos que éste genera en las regiones donde se emplea como pesticida para el control de las enfermedades en la corona, del cuello y de los dedos del banano y plátano.

\section{Referencias}

1. Red Temática VIII - G. Ciencia y Tecnología para el Desarrollo (CYTED). Cooperación Iberoamericana. Eliminación de Contaminantes por Fotocatálisis Heterogénea. Brasil; 2001.p. 316.

2. Arroyave Rojas, J.A. y Correa Ochoa, A.A. Fotodegradación de Malatión usando colector solar. Tesis Pregrado Ingeniería Sanitaria. Universidad de Antioquia; 2001.p. 104.

3. Arroyave R., J. A., Garcés G., L. F. Tecnologías Ambientalmente Sostenibles. Revista de Producción Más Limpia. ISSN 1909 - 0455. Vol 1 No 2 (Julio - Diciembre) 2006. 78 - 86 pg. 
4. Garcés G. L. Cinética de degradación y mineralización del colorante naranja reactivo 84 en aguas. Revista Lasallista de Investigación. 2005(2):21 - 25 .

5. Garcés G. L Et Al. Degradación de aguas residuales de la industria textil por medio de fotocatálisis. Revista Lasallista de Investigación. 2005(2):15-18.

6. Arroyave Rojas. J.A. Et Al. Fotodegradación del Pesticida Mertect empleando Fotofenton con Lámpara de Luz Ultravioleta. Revista Lasallista de Investigación. 2006(2):19 - 24.

7. Arroyave R., J. A., Garcés G., L. F. y Cruz C., A. F. Libro Avances en Investigación Formativa 2008. Capitulo Trabajos de Investigación Terminados, Ciencias Agrarias: Evaluación de la degradación del colorante tartrazina empleando fotocatálisis homogénea con hierro (III) y lámpara de luz ultravioleta. ISBN 978 - 958 - 44 - 6346 - 3. Primera Edición. Medellín, 2010. p.296.

8. Garcés G. L. Et Al. La fotocatálisis como alternativa para el tratamiento de aguas residuales. Revista Lasallista de Investigación. 2004(1): 83 - 92.

9. Arroyave R., J. A., Garcés G., L. F., y Arango R. A Mineralización del colorante Tartrazina mediante un proceso de oxidación avanzada. Revista Lasallista de Investigación. 2009(6): 27 - 34.

10. Arroyave Rojas. J.A. Et Al. Fotodegradación de las aguas residuales con pesticida Mertect en la industria bananera empleando Fotocatálisis con Dióxido de Titanio y Lámpara de Luz Ultravioleta. Revista Lasallista de Investigación. 2007(4): 7 - 13.

11. Arroyave R., J. A., Garcés G., L. F. y Cruz C., A. F. Libro: Fotocatálisis y Electrocoagulación para el tratamiento de aguas residuales: investigaciones y aplicaciones, Capitulo 8: Degradación de las aguas residuales de la industria bananera empleando fotocatálisis con dióxido de titanio y lámpara de luz ultravioleta. Corporación Universitaria Lasallista. ISBN 978-958-98009-3-5. Caldas, Antioquia. 2007.

12. Arroyave R., J. A., Garcés G., L. F. Arango R. A, Agudelo L., C. M. y Martínez R., C. A. Degradación del colorante tartrazina mediante fotocatálisis homogénea empleando lámpara de luz ultravioleta. Revista de Producción Más Limpia. 2008(3):25 - 35.

13. Arroyave R., J. A., Garcés G., L. F. Arango R. A y Agudelo L., C. M. Photo degradation of the tartrazine dye by means of photofenton, using an ultra violet light lamp. Revista Lasallista de Investigación. 2008(5):6 - 12.

14. Arroyave R., J. A., Garcés G., L. F. y Cruz C., A. F. Libro: Fotocatálisis y Electrocoagulación para el tratamiento de aguas residuales: investigaciones y aplicaciones.

15. Arroyave R., J. A., Garcés G., L. F. Arango R. A, Agudelo L., C. M. y Martínez R., C. A. Degradación del colorante tartrazina mediante fotocatálisis heterogénea empleando lámpara de luz ultravioleta. Revista de Producción Más Limpia. 2008(3):21 - 32.

16. Arroyave R., J. A., Garcés G., L. F. y Cruz C., A. F. Libro: Fotocatálisis y Electrocoagulación para el tratamiento de aguas residuales: investigaciones y aplicaciones, Capitulo 7: Fotodegradación del pesticida Mertect empleando fotofenton con lámpara de luz ultravioleta. Corporación Universitaria Lasallista. ISBN 978-958-98009-3-5. Caldas, Antioquia. 2007.

17. Arroyave R., J. A., Garcés G., L. F. y Cruz C., A. F. Libro Avances en Investigación Formativa 2007. Capitulo Trabajos de Investigación Terminados, Ciencias Agrarias: Fotodegradación del Pesticida Mertect empleando fotofenton con Lámpara de Luz Ultravioleta. ISBN 978 - 958 - 44 - 2268 - 2. Segunda Edición. Medellín. 2007.p. 156.

18. Arroyave R., J. A., Garcés G., L. F. y Cruz C., A. F. Libro Avances en Investigación Formativa 2007. Capitulo Trabajos de Investigación Terminados, Ciencias Agrarias: Degradación de las aguas residuales con pesticida Mertect en la industria bananera empleando fotocatálisis con dióxido de titanio y lámpara de luz ultravioleta. ISBN $978-958-44-2268-2$. Segunda Edición. Medellín, 2007. p. 156.

19. Arroyave R., J. A., Garcés G., L. F. y Cruz C., A. F. Libro Avances en Investigación Formativa 2006. Capitulo Trabajos de Investigación en Curso, Medio Ambiente: Fotodegradación del Pesticida Mertect empleando Hierro (III) y Lámpara Ultravioleta. ISBN 978 - 958 - $44-1078-8$. Primera Edición. Medellín, 2007. p.153.

20. Arroyave R., J. A., Garcés G., L. F. y Cruz C., A. F. Libro Avances en Investigación Formativa 2008. Capitulo Trabajos de Investigación Terminados, Ciencias Agrarias: Fotodegradación del pesticida Mertect empleando fotofenton con lámpara de luz ultravioleta. ISBN $978-958$ - 44 - 6346 - 3. Primera Edición. Medellín, 2010.p. 296.

21. Arroyave R., J. A., Garcés G., L. F. y Mejía T., J. Empleo del reactivo de fenton para la degradación del colorante tartrazina. Revista Lasallista de Investigación. 2009(6): 27 - 34.

22. Sygenta Crop. Protection AG. Ficha técnica del Mertect 20 Sl. Edición local comunidad andina. Noviembre 26 de 2002.

23. Vega Gutiérrez, Jesús; Miranda Romero, Alberto, et al. Larva migratoria cutánea. Tratamiento tópico con tiabendazol al 6,25\%. Servicio de Dermatología. Hospital Clínico y Facultad de Medicina de Valladolid, Casos Breves. 2001.p. $171-173$.

24. Muela Pomeda, Susana et al. Efecto de codisolventes y dispersiones sólidas de Polivinilpirrolidona K-30 en la solubilidad del Tiabendazol. En: VI congreso SEFIG y 3as jornadas TF. Departamento de farmacia y tecnología farmacéutica, Universidad de Alcalá; 2003. 85.p. 87.

25. J. Taube, K. Vorkamp; Et Al. Pesticide residues in biological waste. Chemosphere. 2002(49): 1357-1365.

26. Universidad de Antioquia, Facultad Nacional de Salud Pública "Héctor Abad Gómez". Estudio ambiental y de salud por uso de agroquímicos en Urabá - eje bananero. Medellín 1994.p. 50

27. BANATURA. Revista: Programa de gestión social y ambiental del sector bananero Colombiano "manejo de aguas". Medellín. 2003.p.42 - 45.

28. Bermúdez Montoya, L et al. Augura. Revista de Mejores prácticas: tratamiento para el efluente del curado de coronas. Medellín, 2004.
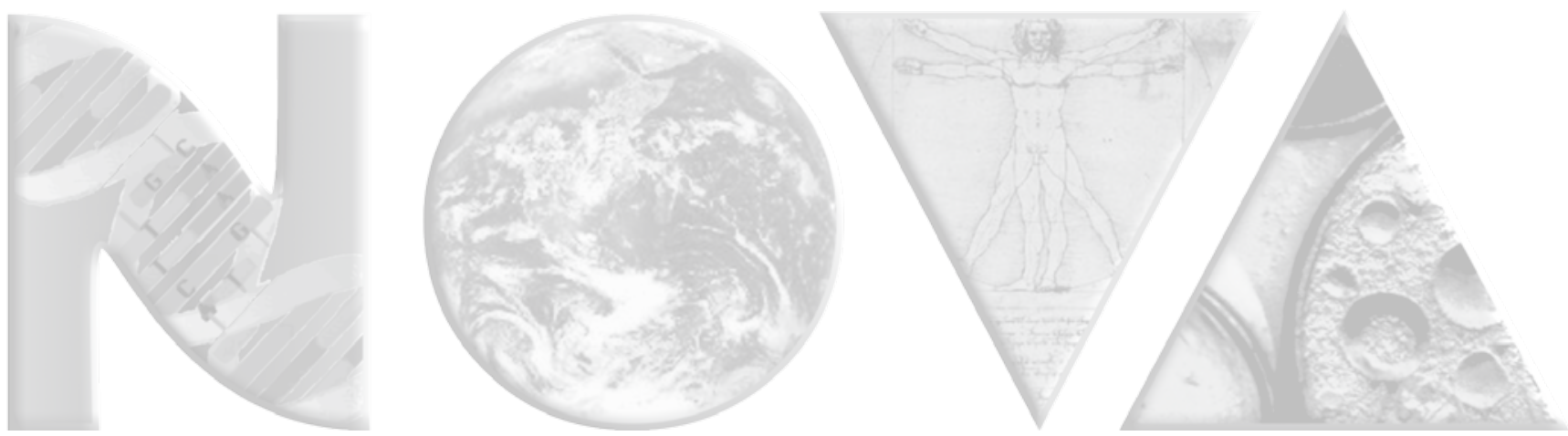